\title{
Severe mental illness increases the risk of death from coronary heart disease or stroke
}

Osborn DPJ, Levy G, Nazareth I, et al. Relative risk of cardiovascular and cancer mortality in people with severe mental illness from the United Kingdom's General Practice Research Database. Arch Gen Psychiatry 2007;64:242-9.

Are people with severe mental health illness at increased risk of death from coronary heart disease, stroke or cancer?

\section{METHODS}

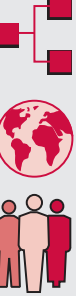

Design: Retrospective cohort study.

Setting: General Practice Research Database (GPRD), covering 741 practices in the UK; June 1987 to April 2002.

으 Population: 46136 people with $\geqslant 1$ diagnosis of severe mental illness (SMI) made after age 18 years and 300426 people who had never been diagnosed with SMI 16 selected randomly for each person with SMI) identified from the GPRD. SMI was defined as bipolar disorder, schizophrenia, schizoaffective disorder, delusional disorder or other non-organic psychoses (identified based on Read and Oxford Medical Information System codes); people with organic psychoses (for example, alcohol, druginduced) were excluded. Only people with at least 6 months' data of sufficient quality were included.

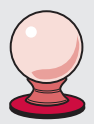

Prognostic factors: Severe mental illness. Hazard ratios (HRs) were calculated using Cox regression, and adjusted for patient age, sex, period in which data were collected (1987-92; 1992-7; 1997-2002), smoking and social deprivation. Analyses were stratified by age because HRs were found to vary significantly by age group.

Outcomes: Deaths from coronary heart disease (CHD), stroke, and the seven most common cancers in the UK (respiratory, colorectal, breast, prostate, stomach, oesophageal and pancreas). Read and OXMIS codes used to search the GPRD for these conditions (and synonyms).

\section{MAIN RESULTS}

Compared to people without SMI, people with SMI were at an increased risk of death from CHD between the ages of 18-49 years (adjusted HR 2.88, 95\% CI 1.77 to 4.70 ), and 50-75 years (adjusted HR 1.76, 95\% CI 1.54 to 2.01 ), but not at 75 years and above (adjusted HR 1.04, 95\% CI 0.91 to 1.18). People with SMI were also at increased risk of death from stroke between the ages of 50-75 years (adjusted HR $1.83,95 \%$ CI 1.45 to 2.31 ), and at 75 years and over (adjusted HR 1.33, 95\% CI 1.16 to 1.52), but not between 18-49 years (adjusted HR 2.39, 95\% CI 0.92 to 6.17). There was no significant association between SMI and risk of death from a common cancer at any age (18-49 years: adjusted HR $1.04,95 \%$ CI 0.60 to 1.82 ; 50-75 years: adjusted HR $1.10,95 \%$ CI 0.93 to 1.29 ; $\geqslant 75$ years: adjusted HR 0.89 , 95\% CI 0.73 to 1.07 ).

\section{CONCLUSIONS}

The risk of mortality from coronary heart disease is increased in people with severe mental illness in the $18-75$ years age group. Mortality from stroke is increased in people with severe mental illness aged 50 years and above. Having a severe mental illness does not increase the risk of death from the seven most common cancers in the UK.

For correspondence: David P J Osborn, PhD, Royal Free and University College Medical School, Rowland Hill Street, London NW3 2PF, UK; d.osborn@medsch.ucl.ac.uk

Source of funding: UK Medical Research Council.

\section{NOTES}

Social deprivation was estimated by general practice neighbourhood and not on individual data. Some smoking data were missing from the GPRD, but sensitivity analyses excluding people with missing data did not differ significantly from the overall analyses. The study also assessed the effects of antipsychotic medication in a secondary analysis (not reported in detail here). In general, it found that CHD and stroke risk was raised even among people with SMI not taking antipsychotics.

\section{Commentary}

ife expectancy among those with severe mental illness lags far behind those without such disorders. Determining the medical conditions -most likely to befall those with mental illness may reveal causes of premature mortality that are amenable to treatment. Most previous studies in this area have had small samples or insufficient follow-up periods.

Osborn and colleagues examined the effects of severe mental illness on mortality rates from cardiovascular disease and cancer using a large national database. The finding of increased risk for coronary heart disease (CHD) and stroke but not for non-respiratory cancer is in accordance with current literature on depression and medical illness. ${ }^{1}{ }^{2}$ It may be that the cardiovascular system is more vulnerable to the physiological effects of mental illnesses than the systems linked to the most common cancers. Alternatively, negative health behaviours common to those with severe mental illness may be linked specifically to heart disease.

Given the risks of premature death from CHD and stroke, those charged with the care of people with severe mental illness should assess and monitor cardiovascular risk and diseases. It is easy for practitioners to see patients as having either a "medical" or a "mental" illness and to focus care in one arena. It is also easy to give up on behavioural risk factor interventions for those with mental illness, because of their reputed resistance to change. However, the predicted riots in response to smokefree in-patient wards never happened, and newer strategies for smoking cessation may yield greater success. Adding interventions to increase healthy behaviours and aerobic exercise periods to the psychoeducational groups and leisure activities that are already standard practice should not be difficult.

We must recognise the risk and remember that mental illness does not occur to the exclusion of other medical problems, but rather in association with them.

Judith A Skala, RN, PhD

Washington University School of Medicine in St Lovis, MO and the United States Department of Veterans Affairs, Albuquerque, NM, USA Competing interests: None.

1 Frasure-Smith N, Lesperance F. Recent evidence linking coronary heart disease and depression. Can J Psychiatry 2006;51:730-7.

2 Irwin M. Depression and risk of cancer progression: an elusive link. J Clin Oncology 2007;25:2343-4. 